International Journal of Pure and Applied Mathematics

Volume 106 No. 1 2016, 157-170

ISSN: 1311-8080 (printed version); ISSN: 1314-3395 (on-line version)

url: http://www.ijpam.eu

doi: 10.12732/ijpam.v106i1.12

\title{
ECCENTRIC CONNECTIVITY INDEX OF SOME CHEMICAL TREES
}

\author{
R.S. Haoer ${ }^{1 \S}$, K.A. Atan ${ }^{2}$, A.M. Khalaf ${ }^{3}$, M. Rushdan ${ }^{4}$, R. Hasni ${ }^{5}$ \\ ${ }^{1,3}$ Department of Mathematics \\ Faculty of Computer Science and Mathematics \\ University of Kufa \\ Najaf, IRAQ \\ ${ }^{1,2,4}$ Institute for Mathematical Research \\ Universiti Putra Malaysia \\ 43400 Serdang, Selangor, MALAYSIA \\ ${ }^{5}$ Department of Mathematics \\ Faculty of Science and Technology \\ University Malaysia Terengganu \\ 21030 UMT Terengganu, MALAYSIA
}

\begin{abstract}
Let $G=(V, E)$ be a simple connected molecular graph. In such a simple molecular graph, vertices represent atoms and edges represent chemical bonds, we denoted the sets of vertices and edges by $V(G)$ and $E(G)$, respectively. If $d(u, v)$ be the notation of distance between vertices $u, v \in V(G)$ and is defined as the length of a shortest path connecting them.Then, the eccentricity connectivity index of a molecular graph $G$ is defined as $\xi(G)=\sum_{v \in V(G)} \operatorname{deg}(v) \operatorname{ec}(v)$, where $\operatorname{deg}(v)$ is degree of a vertex $v \in V(G)$, and is defined as the number of adjacent vertices with $v$. ec(v) is eccentricity of a vertex $v \in V(G)$, and is defined as the length of a maximal path connecting to another vertex of $v$. In this paper, we establish the general formulas for the eccentricity connectivity index of some classes of chemical trees.
\end{abstract}

Received: September 18, 2015

Published: February 3, 2016

${ }^{\S}$ Correspondence author
(C) 2016 Academic Publications, Ltd.

url: www.acadpubl.eu 
AMS Subject Classification: 92E10

Key Words: eccentric connectivity index, eccentricity, chemical trees

\section{Introduction}

A critical step in pharmaceutical drug design continues to be the identification and optimization of compounds in a rapid and cost effective way. The prediction of physic-chemical, pharmacological and toxicological properties of a compound directly from its molecular structure is an important tool in this work. This analysis is known as the study of the quantitative structureactivity relationship (QSAR). In chemistry, a molecular graph represents the topology of a molecule, by considering how the atoms are connected. This can be modelled by a graph, where the points represent the atoms, and the edges symbolize the covalent bonds. Relevant properties of these graph models are then studied, giving rise to numerical graph invariants.

Many such graph invariant topological indices have been investigated. The first one, and most well-known parameter, the Wiener index, was introduced in the late 1940s in an attempt to analyse the chemical properties of alkanes [14]. This is a distance-based index, whose mathematical properties and chemical applications have been widely researched. Numerous other indices have been defined, and more recently, indices such as the eccentric distance sum, and the adjacency cum distance-based eccentric connectivity index have been studied [4], [5], [7]-[13]. These topological indices have been shown to give a high degree of predictability of pharmaceutical properties, and may provide leads for the development of safe and potent anti-HIV compounds. Amendments of some of these indices have also been considered. For example, the augmented eccentric connectivity index $[1,2$ and 6$]$ and the super augmented eccentric connectivity index [3] have been found to be useful indicators in chemical research.

In graph theory, the degree of each vertex represents the valence (number of bonds incident on a vertex) of the molecule in molecular graph. Number of covalent Bonds: 4 for carbon and 1 for hydrogen. If $\operatorname{deg}(\mathrm{v})=1$, then $\mathrm{v}$ is said to be a pendent vertex. An edge incident to a pendent vertex is said to be a pendent edge. A chemical tree is a tree in which the maximal vertex degree does not exceed 4 .

In this paper, we establish the general formulas for the eccentricity connectivity index of some classes of chemical trees. 


\section{Eccentric Connectivity Index of Some Classes of Chemical Trees}

In this section, we construct the general formulas for the eccentric connectivity index of some classes of chemical trees.

Alkanes are hydrocarbons with only single bonds between the atoms, and it has a general formula $C_{n} H_{2 n+2}$, where $\mathrm{n}$ is number of carbon atoms.

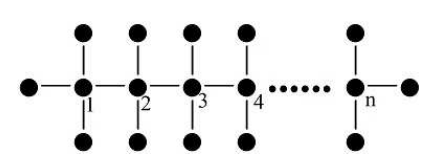

Figure 1: Classes of Alkanes $C_{n} H_{2 n+2}$

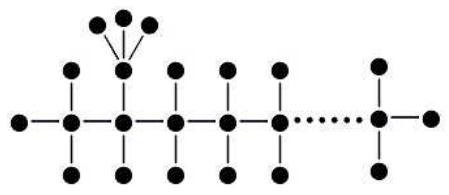

Figure 2: Iso-alkanes (2-methyl alkanes)

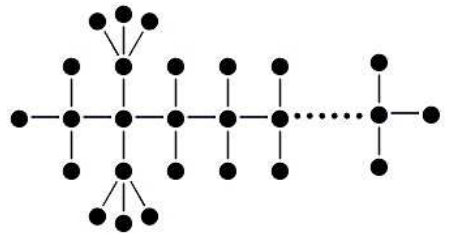

Figure 3: Iso-alkanes (2,2-methyl alkanes)

Theorem 2.1. Let $n$ be number integer positive, then the eccentric connectivity index of a graph $G=C_{n} H_{2 n+2}$ (see Figure 1) is

$$
\xi(G)= \begin{cases}\frac{1}{2}\left(9 n^{2}+14 n+4\right) ; \quad \text { if } n \text { is even; } n \geq 2, \\ \frac{1}{2}\left(9 n^{2}+14 n+1\right) ; \quad \text { if } n \text { is odd } n \geq 1 .\end{cases}
$$

Proof. We will prove by mathematical induction in several cases:

Case 1. If $n$ is even. Let $\mathrm{n}=2$ then $G=C_{2} H_{6}$, whose graph is as follows:

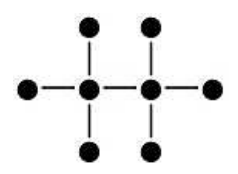


Thus $\xi\left(C_{2} H_{6}\right)=34$. Hence it is true that, $\xi(G)=\frac{1}{2}\left(9 n^{2}+14 n+4\right)$, when $\mathrm{n}=2$. Let $\mathrm{n}=\mathrm{k}$ and $G_{k}=C_{k} H_{2 k+2}$, assume that it is true for $k \geq 2$; $\mathrm{k}$ is even, $\xi(G)=\frac{1}{2}\left(9 n^{2}+14 n+4\right)$.

Construct the graph $G_{k+2}=C_{k+2} H_{2 k+6}$ as follows: The graph $G_{k}$ has the form:

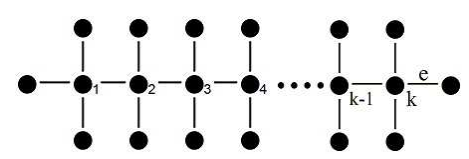

Here $C_{i}$ denoted the position of the carbon vertex at the $i^{\text {th }}$ the position, and e the edge connecting the vertex $C_{k}$ in graph $G_{k}$ with the end hydrogen vertex $\mathrm{H}$.

Let $G^{*}$ be the graph obtained from $G_{k}$ by removing the edge e:

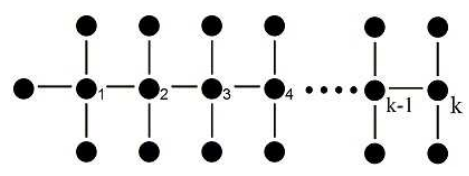

$$
\xi\left(G^{*}\right)=\xi\left(G_{K}\right)-(k+1)=\frac{9}{2} k^{2}+6 k+1 .
$$

Connect the graph $G^{*}$ with the graph $\mathrm{R}$

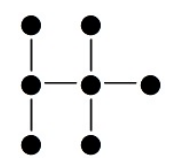

Here the 2 Carbon vertices which is adjacent to 5 hydrogen vertices by connecting $C_{k}$ with $\mathrm{C}$, to obtain the graph $C_{k+2} H_{2 k+6}$ is in the $(k+2)^{t h}$ the position of the carbon vertex of this graph as follows:

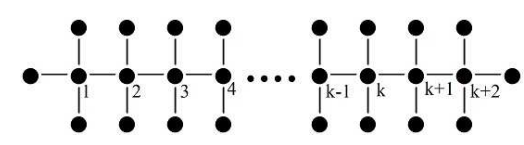

In this case, will get an increase in the eccentricity to $\left(\frac{1}{2} k+1\right)$ of vertices carbon, where $\left(\frac{1}{2} k\right)$ increase by 2 , and one vertex increase by 1 , so the eccentric connectivity index increase is $\left(\frac{1}{2} k\right)(4)(2)+(4)(1)$. Also will get an increase in eccentricity to $\mathrm{k}+1$ of vertices hydrogen by 2 , and just two vertices increase by 1 , so the eccentric connectivity index increase is $(\mathrm{k}+1)(1)(2)+(2)(1)(1)$.

Thus 


$$
\begin{aligned}
& \xi\left(G_{k+2} H_{2 k+6}\right)=\xi\left(G^{*}\right)+\xi(R)+\xi(\text { increases }) \\
=\left(\frac{9}{2} k^{2}+6 k+1\right)+(13 k+25)+\left(\frac{1}{2} k\right)(4)(2)+(4)(1)+(k+1)(1)(2)+(2)(1)(1) & =\frac{9}{2} k^{2}+25 k+34
\end{aligned}
$$

is true when $n=k+2$.

Hence, $\xi(G)$ is true when $\mathrm{n}=2$, also as the assumption that it is true for $\mathrm{n}=\mathrm{k}$ and shown that it is true for $\mathrm{n}=\mathrm{k}+2$, so we have

$$
\xi(G)=\frac{1}{2}\left(9 n^{2}-14 n+4\right)
$$

for all $\mathrm{n}$ is even and $n \geq 2$.

Case 2. If $\mathbf{n}$ is odd. Let $\mathrm{n}=1$ then $G=C_{1} H_{4}$, whose graph is as follows:

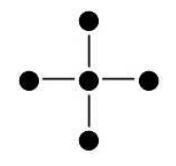

Thus $\xi\left(C_{1} H_{4}\right)=(4)(1)(2)+(1)(4)(1)=12$. Hence

$$
\xi(G)=\frac{1}{2}\left(9 n^{2}+14 n+1\right)
$$

when $\mathrm{n}=1$. Let $\mathrm{n}=\mathrm{k}$ and $G_{k}=C_{k} H_{2 k+2}$. Assume that $n \geq 1 ; \mathrm{k}$ is odd, and

$$
\xi(G)=\frac{1}{2}\left(9 n^{2}+14 n+1\right)
$$
form:

Construct the graph $G_{k+2}=C_{k+2} H_{2 k+6}$ as follows. The graph $G_{k}$ has the

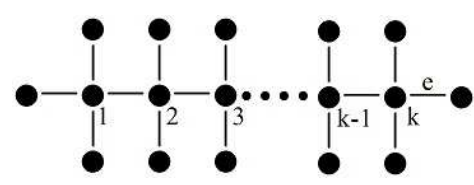

Here $C_{i}$ denoted the position of the Carbon vertex at the $i^{\text {th }}$ the position, and e the edge connecting the vertex $C_{k}$ in graph $G_{k}$ with the end hydrogen vertex H.

Let $G^{*}$ be the graph obtained from $G_{k}$ by removing the edge e: 


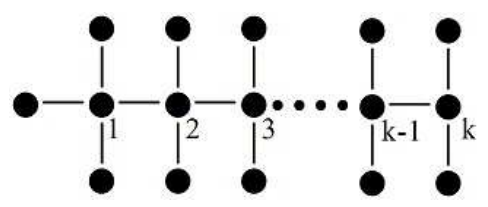

$$
\xi\left(G^{*}\right)=\xi\left(G_{K}\right)-(k+1)=\frac{9}{2} k^{2}+6 k-\frac{1}{2} .
$$

Connect the graph $G^{*}$ with the graph $\mathrm{R}$

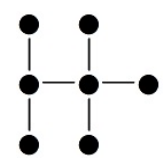

Here the 2 Carbon vertices which is adjacent to 5 hydrogen vertices by connecting $C_{k}$ with $\mathrm{C}$, to obtain the graph $C_{k+2} H_{2 k+6}$ is in the $(k+2)^{t h}$ the position of the carbon vertex of this graph as follows:

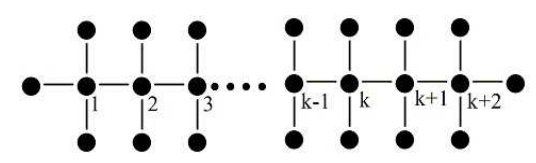

In this case, will get an increase in the eccentricity to $\frac{1}{2}(k+1)$ of vertices carbon, where $\left(\frac{1}{2} k\right)$ increase by 2 , and one vertex increase by 1 ,so the eccentric connectivity index increase is $\frac{1}{2}(k+1)(4)(2)$. Also will get an increase in eccentricity to $\mathrm{k}+2$ of vertices hydrogen by 2 , so the eccentric connectivity index increase is $(\mathrm{k}+2)(1)(2)+(2)$.

Thus

$$
\begin{aligned}
\xi\left(G_{k+2} H_{2 k+6}\right) & =\xi\left(G^{*}\right)+\xi(R)+\xi(\text { increases }) \\
=\left(\frac{9}{2} k^{2}+6 k-\frac{1}{2}\right)+(13 k+25)+\left(\frac{1}{2} k+1\right)(4)(2)+ & (k+2)(1)(2) \\
& =\frac{9}{2} k^{2}+25 k+\frac{65}{2} .
\end{aligned}
$$

is true when $\mathrm{n}=\mathrm{k}+2$.

Hence, $\xi(G)$ is true when $\mathrm{n}=1$, also as the assumption that it is true for $\mathrm{n}=\mathrm{k}$ and shown that it is true for $\mathrm{n}=\mathrm{k}+2$, so we have

$$
\xi(G)=\frac{1}{2}\left(9 n^{2}+14 n+1\right),
$$

for all $\mathrm{n}$ is odd; $n \geq 1$. 
Theorem 2.2. Let $n$ be number integer positive, then the eccentric connectivity index of a graph $G=2-$ methyl alkanes (see Figure 2) is

$$
\xi(G)= \begin{cases}\frac{1}{2}\left(9 n^{2}+8 n-10\right), & \text { if } n \text { is even, } n \geq 4, \\ \frac{1}{2}\left(9 n^{2}+8 n-7\right), & \text { if } n \text { is odd, } n \geq 5 .\end{cases}
$$

Proof. We will prove by mathematical induction in several cases.

Case 1. If $\mathbf{n}$ is even. Let $\mathrm{n}=4$ then $G=C_{4} H_{10}$, whose graph is as follows:

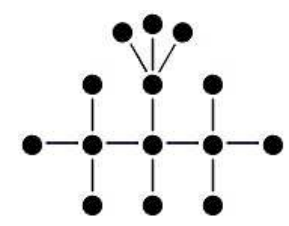

Thus $\xi\left(C_{4} H_{10}\right)=83$. Hence it is true that, $\xi(G)=\frac{1}{2}\left(9 n^{2}+8 n-10\right)$, when $\mathrm{n}=4$. Let $\mathrm{n}=\mathrm{k}$ and $G_{k}=C_{k} H_{2 k+2}$,

Assume that it is true for $k \geq 4$; $\mathrm{k}$ is even, $\xi(G)=\frac{1}{2}\left(9 k^{2}+8 k-10\right)$.

Construct the graph $G_{k+2}=C_{k+2} H_{2 k+6}$ as follows: The graph $G_{k}$ has the form:

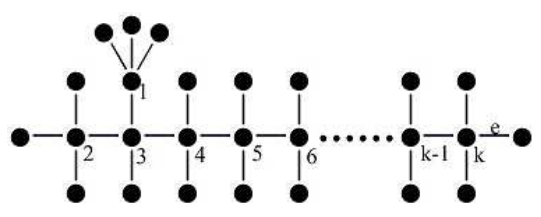

Here $C_{i}$ denoted the position of the carbon vertex at the $i^{\text {th }}$ the position, and e the edge connecting the vertex $C_{k}$ in graph $G_{k}$ with the end hydrogen vertex $\mathrm{H}$.

Let $G^{*}$ be the graph obtained from $G_{k}$ by removing the edge e that is:

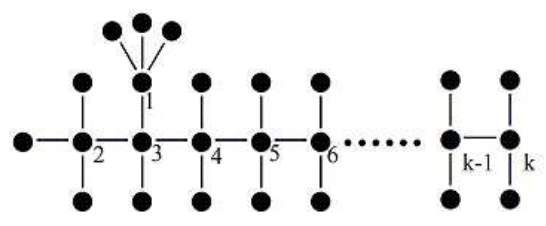

$$
\xi\left(G^{*}\right)=\xi\left(G_{K}\right)-(k)=\frac{9}{2} k^{2}+3 k-5 .
$$

Connect the graph $G^{*}$ with the graph $\mathrm{R}$ 


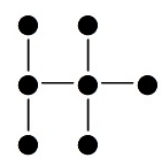

Here the 2 Carbon vertices which is adjacent to 5 Hydrogen vertices by connecting $C_{k}$ with $\mathrm{C}$, to obtain the graph $C_{k+2} H_{2 k+6}$ is in the $(k+2)^{\text {th }}$ the position of the Carbon vertex of this graph as follows:

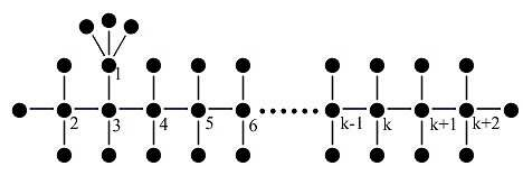

In this case, will get an increase by 2 in the eccentricity $\frac{1}{2}(k+2)$ of carbon atoms,so the eccentric connectivity index of increases is $\frac{1}{2}(k+2)(4)(2)$.

Also will get an increase by 2 in eccentricity for $\mathrm{k}+3$ of hydrogen atoms, so the eccentric connectivity index of increases is $(k+3)(1)(2)$.

Thus

$$
\begin{aligned}
\xi\left(G_{k+2} H_{2 k+6}\right) & =\xi\left(G^{*}\right)+\xi(R)+\xi(\text { increases }) \\
=\left(\frac{9}{2} k^{2}+3 k-5\right)+(13 k+12)+\frac{1}{2}(k+2)(4)(2)+ & (k+3)(1)(2) \\
= & \frac{9}{2} k^{2}+22 k+21 .
\end{aligned}
$$

Is true when $\mathrm{n}=\mathrm{k}+2$. Hence, $\xi(G)$ is true when $\mathrm{n}=2$, also as the assumption that it is true for $n=k$ and shown that it is true for $n=k+2$, so we have

$$
\xi(G)=\frac{1}{2}\left(9 n^{2}+8 n-10\right)
$$

for all $\mathrm{n}$ is even; $n \geq 4$.

Case 2. If $\mathbf{n}$ is odd. Let $\mathrm{n}=5$ then $G=C_{5} H_{12}$, whose graph is as follows:

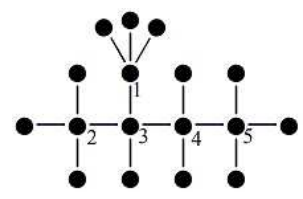

Thus $\xi\left(C_{5} H_{12}\right)=129$. Hence it is true that, $\xi(G)=\frac{1}{2}\left(9 n^{2}+8 n-7\right)$, when $\mathrm{n}=5$.

Let $\mathrm{n}=\mathrm{k}$ and $G_{k}=C_{k} H_{2 k+2}$. Assume that it is true for $k \geq 5$; $\mathrm{k}$ is odd, $\xi(G)=\frac{1}{2}\left(9 k^{2}+8 k-7\right)$.

Construct the graph $G_{k+2}=C_{k+2} H_{2 k+6}$ as follows: The graph $G_{k}$ has the form: 


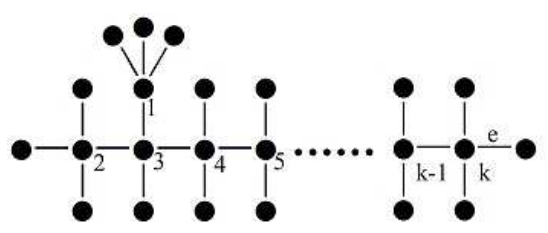

Where $C_{i}$ denoted the position of the Carbon vertex at the $i^{\text {th }}$ the position, and e the edge connecting the vertex $C_{k}$ in graph $G_{k}$ with the end hydrogen vertex $\mathrm{H}$.

Let $G^{*}$ be the graph obtained from $G_{k}$ by removing the edge e that is:

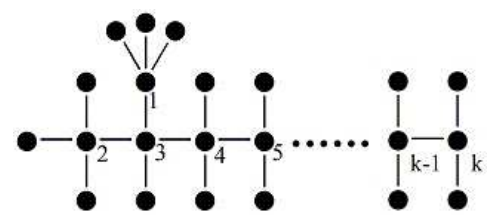

$$
\xi\left(G^{*}\right)=\xi\left(G_{K}\right)-(k)=\frac{9}{2} k^{2}+3 k-\frac{7}{2} .
$$

Connect the graph $G^{*}$ with the graph $\mathrm{R}$ that is:

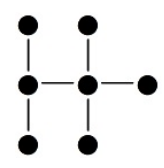

Where the 2 carbon vertices which is adjacent to 5 hydrogen vertices by connecting $C_{k}$ with $\mathrm{C}$, to obtain the graph $C_{k+2} H_{2 k+6}$ is in the $(k+2)^{t h}$ the position of the carbon vertex of this graph as follows:

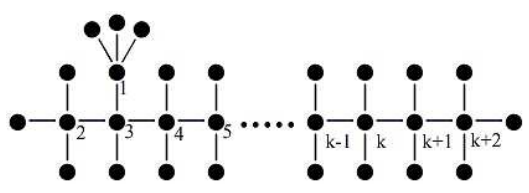

In this case, will get an increase in the eccentricity to $\frac{1}{2}(k+3)$ of carbon atoms, where $\frac{1}{2}(k+1)$ of them increase by 2 , and one vertex increase by 1 ,so the eccentric connectivity index for increases of carbon atoms is $\frac{1}{2}(k+1)(4)(2)+(1)(4)(1)$. Also will get an increase in eccentricity to $k+4$ of hydrogen atoms, where $(k+2)$ of them increase by 2 and two of them by 1 , so the eccentric connectivity index for increases of hydrogen atoms is $(k+2)(1)(2)+(2)(1)(1)$.

Thus

$$
\xi\left(G_{k+2} H_{2 k+6}\right)=\xi\left(G^{*}\right)+\xi(R)+\xi(\text { increases })
$$




$$
\begin{array}{r}
=\left(\frac{9}{2} k^{2}+3 k-\frac{7}{2}\right)+(13 k+12)+\frac{1}{2}(k+1)(4)(2)+(k+2)(1)(2)+(2)(1)(1) \\
=\frac{9}{2} k^{2}+22 k+\frac{45}{2} .
\end{array}
$$

Is true when $\mathrm{n}=\mathrm{k}+2$.

Hence, $\xi(G)$ is true when $\mathrm{n}=1$, also as the assumption that it is true for $\mathrm{n}=\mathrm{k}$ and shown that it is true for $\mathrm{n}=\mathrm{k}+2$, so we have

$$
\xi(G)=\frac{1}{2}\left(9 n^{2}+8 n-7\right)
$$

for all $\mathrm{n}$ is odd; $n \geq 5$.

Theorem 2.3. Let $n$ be number integer positive, then the eccentric connectivity index of a graph $G=(2,2-$ methyl alkanes) (see Figure 3) is

$$
\xi(G)= \begin{cases}\frac{1}{2}\left(9 n^{2}+2 n-24\right), & \text { if } n \text { is even, } n \geq 6, \\ \frac{1}{2}\left(9 n^{2}+2 n-27\right), & \text { if } n \text { is odd, } n \geq 5 .\end{cases}
$$

Proof. We will prove by mathematical induction as follows:

Case 1. If $\mathbf{n}$ is even. Let $\mathrm{n}=6$ then $G=C_{6} H_{14}$, whose graph is as follows:

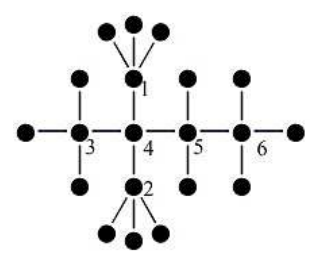

Thus $\xi\left(C_{6} H_{14}\right)=156$. Hence it is true that, $\xi(G)=\frac{1}{2}\left(9 n^{2}+2 n-24\right)$, when $\mathrm{n}=6$.

Let $\mathrm{n}=\mathrm{k}$ and $G_{k}=C_{k} H_{2 k+2}$, assume that it is true for $k \geq 6 ; \mathrm{k}$ is even, $\xi(G)=\frac{1}{2}\left(9 k^{2}+2 k-24\right)$. Construct the graph $G_{k+2}=C_{k+2} H_{2 k+6}$ as follows:

The graph $G_{k}$ has the form:

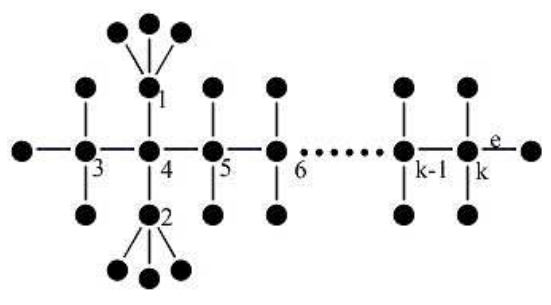


Where $C_{i}$ denoted the position of the carbon vertex at the $i^{\text {th }}$ the position, and e the edge connecting the vertex $C_{k}$ in graph $G_{k}$ with the end hydrogen vertex $\mathrm{H}$.

Let $G^{*}$ be the graph obtained from $G_{k}$ by removing the edge e that is:

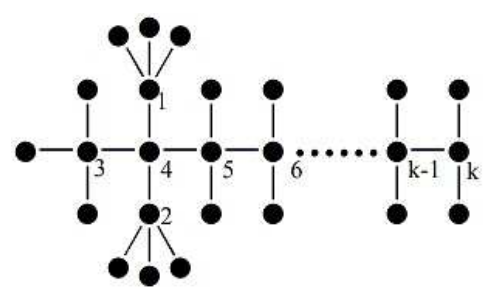

$$
\xi\left(G^{*}\right)=\xi\left(G_{K}\right)-(k-1)=\frac{9}{2} k^{2}-11 .
$$

Connect the graph $G^{*}$ with the graph $\mathrm{R}$

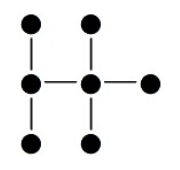

Where the 2 Carbon vertices which is adjacent to 5 hydrogen vertices by connecting $C_{k}$ with $\mathrm{C}$, to obtain the graph $C_{k+2} H_{2 k+6}$ is in the $(k+2)^{t h}$ the position of the carbon vertex of this graph as follows:

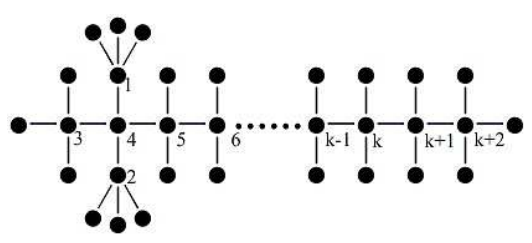

In this case, will get an increase in the eccentricity to $\left(\frac{1}{2} k+2\right)$ of vertices carbon , where $\left(\frac{1}{2} k+1\right)$ increase by 2 , and one vertex increase by 1 , so the eccentric connectivity index of increases is $\left(\frac{1}{2} k+1\right)(4)(2)+(1)(4)(1)$.

Also will get an increase in eccentricity to $(\mathrm{k}+3)$ of vertices hydrogen by 2 ,and just two vertices increase by 1 , so the eccentric connectivity index increases is $(\mathrm{k}+3)(1)(2)+(2)(1)(1)$.

Thus

$$
\begin{aligned}
\xi\left(G_{k+2} H_{2 k+6}\right) & =\xi\left(G^{*}\right)+\xi(R)+\xi(\text { increases }) \\
& =\left(\frac{9}{2} k^{2}-11\right)+(13 k-1)+(4 k+12)+(2 k+8)
\end{aligned}
$$




$$
=\frac{9}{2} k^{2}+19 k+8
$$

Is true when $\mathrm{n}=\mathrm{k}+2$. Hence, $\xi(G)$ is true when $\mathrm{n}=2$, also as the assumption that it is true for $n=k$ and shown that it is true for $n=k+2$, so we have

$$
\xi(G)=\frac{1}{2}\left(9 n^{2}+2 n-24\right)
$$

for all $\mathrm{n}$ is even; $n \geq 6$.

Case 2. If $\mathbf{n}$ is odd. Let $\mathrm{n}=5$ then $G=C_{5} H_{12}$, whose graph is as follows:

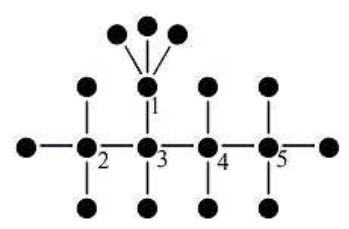

Thus $\xi\left(C_{5} H_{12}\right)=104$. Hence it is true that, $\xi(G)=\frac{1}{2}\left(9 n^{2}+2 n-27\right)$, when $\mathrm{n}=5$. Let $\mathrm{n}=\mathrm{k}$ and $G_{k}=C_{k} H_{2 k+2}$, assume that it is true for $k \geq 5$; $\mathrm{k}$ is odd, $\xi(G)=\frac{1}{2}\left(9 k^{2}+2 k-27\right)$. Construct the graph $G_{k+2}=C_{k+2} H_{2 k+6}$ as follows:

The graph $G_{k}$ has the form:

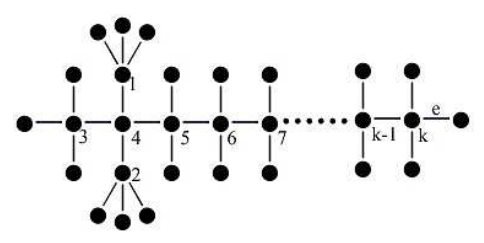

Here $C_{i}$ denoted the position of the carbon vertex at the $i^{\text {th }}$ the position, and e the edge connecting the vertex $C_{k}$ in graph $G_{k}$ with the end hydrogen vertex $\mathrm{H}$.

Let $G^{*}$ be the graph obtained from $G_{k}$ by removing the edge e that is:

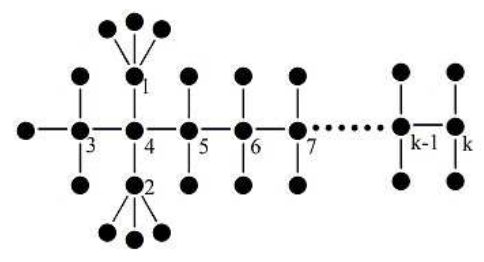

$$
\xi\left(G^{*}\right)=\xi\left(G_{K}\right)-(k+1)=\frac{9}{2} k^{2}-\frac{25}{2} .
$$

Connect the graph $G^{*}$ with the graph $\mathrm{R}$ that is: 


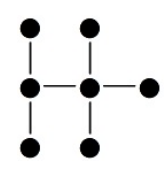

Where the 2 Carbon vertices which is adjacent to 5 hydrogen vertices by connecting $C_{k}$ with $\mathrm{C}$, to obtain the graph $C_{k+2} H_{2 k+6}$ is in the $(k+2)^{t h}$ the position of the carbon vertex of this graph as follows:

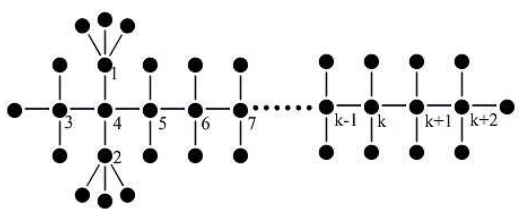

In this case, will get an increase by 2 in the eccentricity to $\frac{1}{2}(k+3)$ of vertices carbon, so the eccentric connectivity index increase is $\frac{1}{2}(k+3)(4)(2)$.

Also will get an increase in eccentricity to $\mathrm{k}+4$ of vertices hydrogen by 2 , so the eccentric connectivity index increase is $(\mathrm{k}+4)(1)(2)$.

Thus

$$
\begin{aligned}
\xi\left(G_{k+2} H_{2 k+6}\right)=\xi\left(G^{*}\right)+\xi(R)+\xi(\text { increases }) & \\
=\left(\frac{9}{2} k^{2}-\frac{25}{2}\right)+(13 k-1)+\frac{1}{2}(k+3)(4)(2)+(k+4)(1)(2) & =\frac{9}{2} k^{2}+19 k+\frac{13}{2}
\end{aligned}
$$

is true when $\mathrm{n}=\mathrm{k}+2$.

Hence, $\xi(G)$ is true when $\mathrm{n}=5$, also as the assumption that it is true for $\mathrm{n}=\mathrm{k}$ and shown that it is true for $\mathrm{n}=\mathrm{k}+2$, so we have

$$
\xi(G)=\frac{1}{2}\left(9 n^{2}+2 n-27\right)
$$

for all $\mathrm{n}$ is odd; $n \geq 5$.

\section{References}

[1] S. Bajaj, S.S. Sambi, S. Gupta, A.K. Madan, Model for prediction of anti-HIV activity of 2-pyridinone derivatives using novel topological descriptor, QSAR and Combinatorial Science 25 (2006) 813-823.

[2] S. Bajaj, S.S. Sambi, A.K. Madan, Topological models for prediction of anti-HIV activity of acylthiocarbamates, Bioorganic and Medicinal Chemistry 13(2005) 3263-3268. 
[3] H. Dureja, S. Gupta, A.K. Madan, Predicting anti-HIV-1 activity of 6-arylbenzonitriles: Computational approach using superaugmented eccentric connectivity topochemical indices, Journal of Molecular Graphics and Modelling 26 (2008) 1020-1029.

[4] H. Dureja, A.K. Madan, Topochemical models for prediction of cyclin-dependent kinase 2 inhibitory activity of indole-2-ones, Journal of Molecular Modeling 11(2005) 525-531.

[5] H. Dureja, A.K. Madan, Topochemical models for the prediction of permeability through blood-brain barrier, International Journal of Pharmaceutics 323 (2006) 27-33.

[6] H. Dureja, A.K.Madan, Predicting anti-HIV activity of dimethylaminopyridin-2-ones: Computational approach using topochemical descriptors, Chemical Biology and Drug Design 73 (2009) 258-270.

[7] S. Gupta, M. Singh, A.K. Madan, Application of graph theory: Relationship of eccentric connectivity index and Wieners index with anti-inflammatory activity, Journal of Mathematical Analysis and Applications 266 (2002) 259-268 http://dx.doi.org/10.1006/jmaa.2000.7243.

[8] V. Kumar, A.K. Madan, Application of graph theory: Prediction of glycogen synthase kinase-3 beta inhibitory activity of thiadiazolidinones as potential drugs for the treatment of Alzheimers disease, European Journal of Pharmaceutical Sciences 24 (2005) 213-218.

[9] V. Kumar, A.K. Madan, Application of graph theory: Prediction of cytosolic phospholipase A(2) inhibitory activity of propan-2-ones, Journal of Mathematical Chemistry 39 (2006) 511-521.

[10] V. Kumar, A.K. Madan, Application of graph theory: Models for prediction of carbonic anhydrase inhibitory activity of sulfonamides,Journal of Mathematical Chemistry 42 (2007) 925-940.

[11] V. Lather, A.K. Madan, Application of graph theory: Topological models for prediction of CDK-1 inhibitory activity of aloisines, Croatica Chemica Acta 78 (2005) 55-61.

[12] S. Sardana, A.K. Madan, Predicting anti-HIV activity of TIBO derivatives: A computational approach using a novel topological descriptor, Journal of Molecular Modeling 8 (2002) 258-265.

[13] V. Sharma, R. Goswami, A.K. Madan, Eccentric connectivity index: A novel highly discriminating topological descriptor for structure-property and structureactivity studies, Journal of Chemical Information and Modeling 37 (1997) 273-282.

[14] H. Wiener, Structural determination of the paraffin boiling points, Journal of the American Chemical Society 69 (1947) 17-20. 